\title{
Breves Anotaciones Sobre Recientes Conceptos en Anticoncepción
}

\author{
Dr. Antonio Lomanto Morán*
}

Todo médico, aunque no admita moralmente el principio de la limitación de la natalidad, debe estar al corriente de los métodos que utilizan millones de familias del mundo entero para limitar su descendencia

Posiblemente no existe en nınguna rama de la medicina, tan necesario $y$ al mismo tiempo tan controvertido, el tema que vamos a tratar.

Con la tecnología moderna hemos alcanzado un grado de seguridad y eficacia nunca antes logrado, por lo tanto la descripción de los procedimientos anticonceptivos se llevará a cabo, teniendo en cuenta ese criterio.

* Jefe de la Sección de Ginecología y Pregrado Facultad de Medicina, Universidad Nacional de Colombia.

Jefe de la Sección de GinecologíaClínica. Hospital Fray Bartolomé de Las Casas. Caja de Previsión Distrital.

\section{METODOS DE BARRERA}

Es difícil evaluar la efectividad, ya que el problema principal no es el fracaso del producto en sí, sino una falla de la usuaria en la correcta aplicación del producto y sobre todo, en su empleo constante. Los investigadores demuestran que las pacientes, en estudios efectuados en clínicas pequeñas dirigidas por médicos particulares, tienen tasas de 1 a 5 embarazos por 100 años-mujer (1).

Sin lugar a dudas la contracepción de barrera ofrece ventajas a las parejas, que podemos resumir en los siguientes puntos:

1. La elección del método se puede realizar en privado.

2. No es indispensable el control del médico.

3. Protege en una incidencia considerablemente alta contra las enfermedades venéreas.

4. Algunas investigaciones sugieren menor incidencia de neoplasias intra- 
epiteliales cervicales en las mujeres que lo usan.

\section{ESPERMICIDAS}

Tienen más aceptación en los países más desarrollados, en donde se utilizan cerca del $3 \%$ en el grupo de mujeres fértiles (2). Se ha resumido el mecanismo de acción de los espermicidas en los siguientes factures.

- Disminuyendo la movilidad del espermatozoide.

- Inactivando las enzimas necesarias para que el espermatozoide penetre en el óvulo.

- Capacidad para actuar como barrera física en el cervix para prevenir la penetración del espermatozoide.

En conclusión podemos decir, que el espermicida ideal debe ser aquel que actúe como barrera física y que es capaz de penetrar en el lago seminai para inactivar el espermatozoide.

Los ingredientes activos de superficie más utilizados, son el nonoxinol 9 y el mefengol. Tienen la facultad de romper la capa externa de lipoproteínas del espermatozoide, dejando que escapen componentes intracelulares tales como el citocromo y otras proteínas necesarias (3).

Es importante mencionar los inhibidores enzimáticos que atacan la proacrosina, la hialuronidasa y enzimas que son necesarias para que el espermatozoide fertilice el óvulo. El Gossy pol, derivado de las semillas del algodón, es uno de estos agentes, ha sido asociado con la polivinil-pirrolidona con la cual alcanza una inactividad del esperma.

Recientemente se ha experimentado con el propanol con 80 mgrs, cuya tasa de embarazo calculado en un año de vida fue de $3.4 \%$ mujeres (4).

\section{ANILLOS VAGINALES}

Las diferentes organizaciones internacionales están investigando con anillos hechos de goma y silicona para utilizarlos intravaginalmente durante 30 días, liberando $5 \mathrm{mg}$ de nonoxinol 9 por día. Aún no hay datos que ameriten su comercialización (5).

\section{ESPONJ AS VAGINALES}

Recientemente se ha investigado sobre una esponja hecha de colágeno que tiene una capacidad absortiva considerable. En consideración a la alta incidencia de fracasos (25응 a 35 을 no ha tenido mayor acep tación.

También se está investigando en la esponja de colatex, elaborada con poliuretano impregnada de nonoxinol 9. Se ha comercializado en Estados Unidos y últimamente Holanda, Noruega y el Reino Unido (6).

\section{DIAFRAGMA}

Artefacto poco utilizado en nuestro medio, dadas las condiciones mínimas para su utilización. Tiene más aceptación en los países más desarrollados. Ha descendido su empleo por el advenimiento de métodos con una eficacia mayor. Referente a la efectividad se mencionan estadísticas que alcanzan fracasos en el primer año hasta $23 \%$ por 100 usuarias. 


\section{CAPUCHONES}

Fueron tan poco populares como el diafragma, elaborados a base de latex. No ha sido posible obtener un criterio definido sobre las técnicas de uso.

Los métodos de barrera pueden ser los de elección para las parejas con las siguientes características:

1. Aquellas en quienes existe contraindicación, o no pueden tolerar los orales o los dispositivos intrauterinos.

2. Las que se encuentran en el postparto o en período de lactancia.

3. Personas sexualmente activas, con cierto número de compañeros.

4. Aquellas que por diferentes motivos no pueden acudir al médico.

5. Usuarias que se encuentran en período de "descanso" de otros anticonceptivos.

6. Aquellas que olvidan la píldora y desean más seguridad.

7. Rechazo a otros procedimientos anticonceptivos.

8. Cuando se desea lograr un ciento por ciento de efectividad, si usan el DIU.

9. Eyaculadores prematuros, escogen el condón para disminuir la sensación.

Se han mencionado como contraindicaciones para los métodos de barrera, pacientes con prolapsos, reacción alérgica a los medicamentos y la insuficiente motivación al método.

\section{DISPOSITIVOS INTRAUTERINOS}

Después de aproximadamente dos décadas de uso, los dispositivos intrauterinos, (DIU), continúan siendo una técnica de control de nacimientos, generalmente efectiva, segura y útil.

A diferencia de los anticonceptivos orales que actúan en todo el organismo, los efectos del DIU se limitan al aparato genital.

Aproximadamente el 5\% de las mujeres en edad fértil en los países en desarrollo, es el método de elección. A nivel mundial aproximadamente 60 millones de mujeres lo están usando, de los cuales 40 millones se utilizan en China y 15 millones en el resto del mundo.

\section{TIPOS DE DIU}

La producción comercial de artefactos intrauterinos es en realidad considerable, pero en la actualidad los más utilizados son: el anillo de plástico impregnado en bario (lippes), el 7 con cobre en su extensión axial, la $\mathrm{T}$ de cobre 200 y finalmente un dispositivo que contiene levonorgestrel (progestasert). El escudo cuya producción se inició hacia el año de 1974 (8) ya fue descontinuado por la incidencia tan alta de sepsis, posiblemente por su cola multifilamentosa.

La $\mathrm{T}$ con un componente de $380 \mathrm{mcg}$ de cobre salió al comercio pero por razones aún no determinadas fue retirada (9), (23).

Sin embargo, y a pesar de las experiencias extensas y de la permanente investigación que se realizó tanto. en países desarrollados como en vías de desarrollo, algunos de los problemas que plantea 
el uso del dispositivo permanece sin resolver, y algunas soluciones nuevas y prometedoras aún no han sido comprobadas.

Los problemas que plantea el uso del DIU son:

1. Menstruaciones más abundantes o pequeñas manchas o pérdida intermenstrual.

2. Expulsión rápida después de su inserción.

3. Infecciones pélvicas con mayor frecuencia.

4. Embarazos indeseados que tienen mayores probabilidades de ser ectópicos o que conduzcan a un aborto séptico, que en el caso de embarazos en no usuarias.

Las experiencias con el DIU a través del tiempo, han demostrado, que dentro de ciertos límites, ciertos factores clínicos e individuales podrían tener mayor impacto en la continuación del uso que el diseño del DIU por sí mismo.

Los prometedores nuevos enfoques que podrían eliminar o minimizar algunos de estos problemas incluyen:

1. Dispositivos medicados que son efectivos por más de 10 años.

2. Dispositivos elaborados con características que reduzcan la pérdida sanguínea.

3. Dispositivos diseñados en tal forma que sea menor la incidencia de expulsión principalmente en el post-parto y post aborto.
4. Perfeccionamiento adaptativo del tamaño y configuraciones del DIU al útero.

5. Mejoras en los instrumentos y en las técnicas de inserción.

\section{Mecanismos de Acción}

En la actualidad se acepta que la acción contraceptiva del DIU se debe a la producción de una reacción inflamatoria local estéril producida por la presencia del cuerpo extraño en el útero. La adición de cobre aumenta la reacción inflamatoria. Se ha comprobado que la fase corta del transporte de esperma desde el cervix hasta el oviduc to está marcadamente disminuida en mujeres que tienen el DIU. Hay un incremento de casi $1000 \%$ en el número de leucocitos existentes en los lavados uterinos de la cavidad endometrial- humana 18 semanas después de la inserción (10).

La vida media del progestasert es de un año mientras que la $\mathrm{T}$ de cobre 200 es de 2 años.

\section{Fechas de Inserción}

Es costumbre que el DIU se debe aplicar durante o recién pasada la menstruación. Las razones que se aducen son en primer lugar porque el cervix está teóricamente abierto, segundo el sangrado menstrual enmascara el producido por la aplicación del artefacto. Cuando las candidatas están ingiriendo anticonceptivos orales se puede aplicar en cualquier fecha del ciclo.

Es importante tener el estudio citológico previo a la inserción. 
Con relación a la aplicación postparto se sugiere hacerlo 2 a 3 meses después con el fin de disminuir la alta incidencia de expulsión que se ha comprobado.

\section{Efectos Adversos}

Está demostrado que durante el primer año de uso es cuando se observan el mayor número no sólo de expulsiones sino de embarazos. Se dice que aproximadamente el $15 \%$ del DIU son retirados durante el primer año de uso (11).

La principal causa de remoción del DIU es la hemorragia, siendo un factor importante el criterio para la escogencia del artefacto.

No está establecido todavía con exactitud la causa del sangrado. La perforación es otra posibilidad durante la aplicación del DIU y está íntimamente relacionada con el tipo y la fuerza empleada durante la inserción. Cuando se sospecha perforación se deben utilizar .todos los mecanismos para extraerlo ya sea por laparoscopia o por laparotomía. Hasta hoy no existen evidencias de aumento en la incidencia de malformaciones congénitas de productos de mujeres que tenían el DIU (12).

En todas las series de embarazos con DIU se comprobó una mayor frecuencia de abortos. En aquellos casos en los cuales queda embarazada una mujer con el DIU, lo ideal es retirarlo, si es posible hacerlo, con el fin de descender la frecuencia de prematurez. En caso de ser retirado se le debe advertir a la paciente la alta incidencia de abortos. La infección es otra de las posibles complicaciones que se pueden presentar posteriormente a la inserción del DIU. Estadísticamente se há demostrado un aumento de la en- fermedad pélvica inflamatoria (EPI). En los casos en que se compruebe una EPI, se debe hacer el tratamiento médico inicial y una vez esté dominado practicar la remoción del DIU.

En consideración a los mecanismos de acción del DIU y teniendo en cuenta que la reacción inflamatoria también se produce en las trompas, se afirma que cuando una mujer queda embarazada con un DIU, se debe sospechar inicialmente un embarazo extrauterino.

No existen estudios de ninguna índole que relacionen el uso del DIU con cualquier tipo de CA del aparato genital.

\section{Contraindicaciones}

Cuando se tienen en cuenta las contraindicaciones de los DIU, seguramente tendremos menos efectos adversos. Entre estas se cuentan: sospecha de embarazo, infecciones pélvicas agudas, tumores tanto del cuello como del cuerpo uterino, cardiopatías vasculares, antecedentes de hemorragias no aclaradas y finalmente pacientes que están utilizando anticoagulantes (13).

\section{HORMONALES}

Las ventajas de la utilización de los anticonceptivos orales abarcan toda una gama de preocupaciones por la salud, ocupando el primer lugar entre ellas, como es natural, la eficacia.

Las encuestas realizadas revelan que la píldora es el método reversible más. eficaz para prevenir un embarazo no deseado. En comparación los dispositivos jntrauterinos tienen en un período de 12 meses una tasa de fallas del doble de la 
píldora. Los condones del triple, los diafragmas del cuádruple y demás del quíntuple y los otros métodos. Naturalmente la eficacia de los anticonceptivos orales depende de su uso regular.

De los efectos ventajosos que algunas mujeres derivan del uso de los anticonceptivos orales figuran los siguientes:

1. Protección contra Enfermedad Inflamatoria Pélvica (EIP). Las actuales usuarias de anticonceptivos orales enfrentan la mitad del riesgo de las no usuarias y la cuarta parte del riesgo de las usuarias del dispositivo intrauterino.

2. Protección contra el embarazo ectópico. Las actuales usuarias de anticonceptivos orales corren la décima parte del riesgo de las no usuarias, de tener embarazos ectópicos.

3. Protección contra el cáncer de endometrio. Las mujeres que utilizan los anticonceptivos orales corren la tercera parte del riesgo de las que nunca los usaron de padecer este tipo de cáncer.

4. Protección contra el cáncer de los ovarios. En comparación con las que nunca usaron los anticonceptivos orales, aquellas que los han usado corren dos tercios del riesgo de tener cáncer de ovario.

5. Protección contra enfermedad benigna de las mamas. Las actuales usuarias tienen en comparación con las no usuarias, la mitad menos de frecuencia de quistes de mamas.

6. Eliminación de una serie de trastornos menstruales comunes, inclusive menstruaciones irregulares, tensión premenstrual e hipermenorrea.

\section{MECANISMOS DE ACCION}

1. Los anticonceptivos orales evitan la ovulación al interferir en el hipotálamo la liberación del factor de hormona luteinizante.

2. Modifican el endometrio en tal forma que disminuye la producción de glucógeno por las glándulas endometriales $y$ se cuenta con menor substrato energético para que sobreviva el blastocisto en la cavidad uterina.

3. Modifican las concentraciones del ácido siálico del moco cervical tornándose más espeso, más viscoso y de menor volumen y con ello retardan la penetración del espermatozoide.

4. Alteran la capacidad de respuesta del ovario a la estimulación por las gonadotropinas.

5. También se esboza la posibilidad de alterar la motilidad de las trompas y con ello el transporte del óvulo y del espermatozoide (14).

\section{TIPOS DE CONTRACEPTIVOS}

1. La píldora trifásica: en un intento de disminuir la dosis hormonal por ciclo, se ha creado la formulación trifásica, que remeda el ciclo menstrual normal.

A pesar de la drástica disminución de la cantidad total de hormonas por ciclo (30 por 100 menor de la de los microdosificados tipo 30/150), la eficacia anticonceptiva es la misma que la de los preparados estándar, consiguiéndose una reducción significativa en la aparición de efectos secundarios.

Otra ventaja para las jóvenes usuarias consiste en que con la formulación tri- 
fásica la reducción en la secreción de $\mathrm{FSH}$ y $\mathrm{LH}$ es menos intensa que con las formulaciones estándar. Sin embargo, la función gonadal presenta una inhibición gonadal similar a la obtenida con los anticonceptivos orales combinados clásicos.

2. Implantes: Consiste en la aplicación de seis aditamentos que contienen cada uno 36 miligramos de levonorgestrel en el brazo por medio de una incisión de $3 \mathrm{~mm}$. Según las últimas estadísticas tiene una tasa de fallas del $1 \%$ (mujeres-años); como efectos colaterales más frecuentes se mencionan hipermenorreas y metrorragias. La efectividad-vida es de 5 años (15).

3. Combinados: Según el índice de Pearl se obtiene el $0.1 \%$ de fracasos. Tiene una combinación de estrógenos y progestágenos y es el más utilizado en el orbe.

4. Minipíldora: Como dato favorable al no poseer estrógenos tiene menos efectos colaterales. Debido a la baja composición hormonal se presentan manchados intermenstruales.

5. Gestágenos de depósito: Se ha comprobado que con una sola aplicación de dosis altas del progestágeno alcanza la anticoncepción desde 1 hasta 6 meses. En consideración a los efectos colaterales no ha sido aprobado por los organismos de salud de los países desarrollados (16).

6. Píldora postcoital: Son medicamentos a base de estrógenos y estrógenos con progestágenos. Es importante anotar que debe ser administrado hasta las 72 horas posteriores a las relaciones en dos tomas cada 12 horas. Es el método, ideal cuando hay pocas relaciones o en los casos de violación.

7. La $T$ de progesterona: $E s$ el único dispositivo hormonalmente activo que se distribuye en el comercio en la actualidad. Libera un promedio de 65 microgramos de norgestrel. La vida media es de un año.

Los métodos secuenciales han sido retirados del comercio por sus efectos colaterales así como los que actualmente se investigan como es el caso de los inmunizadores contra HCG y también los anillos vaginales.

\section{EFECTOS EN EL APARATO REPRODUCTOR}

La ingestión cíclica de anticonceptivos ocasiona una secreción prematura, seguida de una regresión que precede a la atrofia en el endometrio. Está demostrado que cuando se administran anticonceptivos con componente estrogénico, aumentan las neoformaciones del útero.

Los ovarios de mujeres que usan anticonceptivos son muy similares a los de la menopáusica, con características que ceden al suspender la terapia (17).

Las alteraciones a nivel del cuello uterino como es el caso de la hiperplasia polipoide no tiene relación alguna con entidades malignas.

Hay publicaciones acerca de la presencia de moniliasis en las mujeres que ingieren anticonceptivos, casos en los cuales se sugiere una curva de tolerancia a la glucosa (18).

\section{EFECTOS COLATERALES}

Las usuarias de anticonceptivos por mucho tiempo tienen una mayor frecuen- 
cia de pruebas anormales de tolerancia a la glucosa. Hay una gran posibilidad de que los anticonceptivos produzcan las mismas alteraciones en el metabolismo de glucosa que aparecen en las embarazadas.

Las usuarias de anticonceptivos tienen más posibilidad de trastornos de la coagulación cuando existen factores de riesgo como son la hipertensión, obesidad, diabetes y tabaquismo.

Los factores de la coagulación están alterados, principalmente el I, el II, IV, VIII y el XI, la tolerancia a la heparina y la agregación plaquetaria. Esta es la razón para contraindicar los anticonceptivos cuando hay trastornos de la coagulación.

\section{CONTRAINDICACIONES}

Según la Organización Mundial de la Salud se consideran como contraindicaciones absolutas, las siguientes:

1. Neoplasias estrógenas dependientes.

2. Alteraciones de la función hepática.

3. Trastornos de la coagulación.

4. Anomalías de la menstruación no diagnosticadas.

5. Embarazo.

6. Disrritmias cerebrales.

\section{ANTICONCEPTIVOS Y CANCER}

En el estudio realizado por la Asociación de Ginecólogos de los Estados Unidos de Norteamérica, se comprobó que no existe relación alguna entre el uso de anticonceptivos orales y cáncer de los órganos de la reproducción.

\section{RECOMENDACIONES PARA LA PRESCRIPCION}

Siendo los anticonceptivos medicamentos muy activos y con riesgos que ya han sido mencionados, antes de la prescripción deben tenerse en cuenta los siguientes factores:

1. Exclusión de las contraindicaciones por anamnesis y examen físico.

2. Exámen ginecológico y citológico.

3. Exploración minuciosa de las mamas.

4. Dosificación de la glicemia.

5. Evaluación de las cifras tensionales.

\section{EFICACIA}

Los anticonceptivos orales son los más aceptados entre todos los métodos de control de la natalidad por la eficacia y la conveniencia que ofrecen, no obstante los riesgos que implican.

Como se dijo anteriormente, la terapia combinada es la más efectiva dentro del grupo, pues tiene una tasa de fracasos del $0.1 \%(19)$.

\section{ESTERILIZACION FEMENINA}

La esterilización femenina voluntaria constituye el método anticonceptivo más utilizado a nivel mundial. La rápida propagación de los métodos quirúrgicos se ha hecho posible gracias a la alta eficacia que ofrecen. Según los organismos más autorizados de planificación familiar, alrededor de 95 millones de mujeres están 
en la actualidad con este procedimiento anticonceptivo.

En el presente artículo me limitaré a hacer mención solamente de los procedimientos quirúrgicos más utilizados.

De las primeras informaciones figura la técnica descrita por Madlener, consistente en el aplastamiento de la base del asa antes de la ligadura con atrofia subsiguiente de la misma. El índice de fracasos de esta técnica alcanzó el $14 \%$, atribuida posiblemente al hecho de emplear material no absorbible.

A fines de 1924 Irving introduce el muñón proximal en la pared muscular del útero y el distal entre las hojas del ligamento ancho. Esta técnica presentó como era de esperarse problemas de sangrado intraoperatorio.

Posiblemente la técnica más difundida mundialmente es la descrita por Pomeroy en 1939, consistente en practicar una sutura simple para anudar el asa tubárica a nivel del tercio medio, después de la cual es resecado. El material de sutura es absorbido rápidamente, reduciendo de esta manera las posibilidades de recanalización, ya que los extremos seccionados de las trompas se separan.

La fimbriectomía de Kroener descrita hacia el año de 1935. Después de esta técnica fue muy poco lo que se obtuvo en el campo de la esterilización.

Uchida describe en 1975 una técnica en la cual después de infiltrar con solución salina el ligamento ancho, reseca específicamente el trayecto muscular de la trompa en un sector de 3 a $4 \mathrm{cms}$. Peritonizado posteriormente.
Infortunadamente ningún autor informa sobre esta técnica y Uchida sobre 20.000 casos reporta éxito en el total de los casos (20). La elección del método de esterilización debe hacerse en su eficacia, ésto en su grado de irreversibilidad.

\section{LAPAROSCOPIA}

A comienzos de 1960 se iniciaron los primeros procedimientos laparoscópicos. Inicialmente se utilizó la electro coagulación, que consiste en utilizar corriente eléctrica de alta frecuencia para coagular y obstruir las trompas. Es una técnica segura pero tiene el riesgo de quemar órganos vecinos. La termocoagulación destruye la trompa en el lugar de los circuitos eléctricos. Con este procedimiento al parecer no se producen quemaduras a órganos vecinos. El uso de la termocoagulación no se ha generalizado mucho y no ha sido posible establecer su eficiencia.

El dispositivo mecánico más difundido a nivel mundial es el anillo tubárico, conocido también con el nombre de banda elástica o anillo de Yoon, desarrollado en la Facultad de Medicina de la Universidad de Johns Hopkins en 1973.

La recanalización de la trompa es más factible con esta técnica que con la electrocoagulación o las técnicas de ligaduras más complejas. La técnica es relativamente sencilla en personal bien entrenado (21).

VENTAJAS:

- Baja tasa de complicaciones.

- Puede realizarse en corto tiempo.

- La incisión es pequeña. 
- El equipo se puede utilizar para procedimientos diagnósticos.

- Menos dolor que los otros procedimientos.

- Ideal para campañas masivas de planific ación.

\section{DESVENTAJAS:}

- Las complicaciones son infrecuentes pero de gran magnitud.

- Debe realizarla un Gineco-obstetra o cirujano abdominal.

- Es una técnica difícil de aprender.

- La insuflación ocasiona dolores principalmente en los hombros.

- El equipo es costoso así como su mantenimiento.

- No está indicada en el post-partum.

\section{CONTRAINDICACIONES}

Cuando las pacientes tienen patologías cardiopulmonares severas, está contraindicada la laparoscopia por la exagerada insuflación de gas intraabdominal y la mayor absorción de gas carbónico, pueden ocasionar alteraciones respiratorias graves. La obesidad exagerada constituye una contraindicación por la dificultad para introducir la aguja con el propósito de hacer el pneumoperitoneo.

Cuando existen antecedentes de peritonitis, de causa no bien establecida, también es una contraindicación, pues hay la posibilidad de encontrar asas intestinales adheridas a la pared abdomi- nal que pueden ser lesionadas durante la introducción del trocar.

Según un informe obtenido del Consejo de Población en marzo de 1986 y sobre un total de 236.858 laparoscopias, la incidencia de fracasos asciende a 3.7 por mil.

\section{MINILAPAROTOMIA}

La minilaparotomía es una técnica sencilla y segura. Puede realizarse en cualquier momento, incluido el postparto o después de un aborto. La simplicidad, seguridad y eficacia, hacen de ella el método ideal, con laparoscopia, el método irreversible más seguro hasta ahora descrito. Debido a que la incisión es más pequeña, la minilaparotomía tiene menor número de complicaciones que técnicas similares.

VENTAJAS:

- Fácil de aprender.

- Puede ser practicada por cualquier médico con experiencia quirúrgica.

- Se pueden utilizar equipos quirúrgicos poco costosos.

- Las complicaciones son menores.

- Puede realizarse en el postparto.

DESVENTAJAS:

- Es una intervención un poco más larga que la laparoscopia.

- Difícil en mujeres obesas y con adherencias post-operatorias.

- La cicatriz es pequeña pero visible. 
- Dolor abdominal en más del $50 \%$ de las pacientes.

- Tasa mayor de infección.

En relación con las contraindicaciones son similares que para la laparoscopia. Según las publicaciones recientemente obtenidas de los organismos internacionales de planificación familiar, aunque inferiores a la laparoscopia los fracasos ascienden a 4.5 por mil (22).

Ultimamente ha surgido mucha preocupación sobre las técnicas que por sus

\section{BIBLIOGRAFIA}

1. BOUNDS, W. Recent advances in barrier methods of contraception. Novum, 23. 6-7. 1983.

2. CAMERON, S.M., WALTER, D.P., \& ZANEVELD, L.J.D. Vaginal spermicidal activity of gossypol in the Macaca acritiodes. Fertility and Sterility. 37, 273. 1982.

3. FELDBLUM. P. (Family Health International) (Update data from International Trial of contraceptive Sponge) personal comunication. November 4. Reported in new Development in vaginal contraception, 1984. Population reports series H., Number 7, 1983.

4. ZIPPER, J. WHELER, R.G., POTTS, D.M. \& RIVERA. M., propanolol as a novel, effective espermicide: preliminary finding. Brithis Medical Journal, 287, 1245-1246, 1983. características pueden permitir en un futuro la recanalización de las trompas cuando se desee otro embarazo.

El concepto general al respecto se limita a puntualizar que es necesario dejar un mínimo de $5 \mathrm{cms}$ de trompa para poder realizar el procedimiento con éxito.

Antes de la esterilización es necesario que se realice un adecuado asesoramiento a ambos conyúges y que la decisión no sea apresurada.

5. HUGGINS, G., VESSEY, M. FLAVEL, R. et al. Vaginal spermicides and outcome of pregnancy: fidings on a large study. Contraception 25 (3), 219-230, 1983.

6. KENNEDY, W.P., VAN DER VEN, H. H., WALLER, D.P. Gossypol inhibition of oocyte penetration and sperm acro$\sin$. Biology of reproduction, 26 (supplement 1), 18a. 1982.

7. McCLURE, Z. Failure of contraceptive methods. Family planing information Service, 16, 59-61. (1981).

8. JAIN, A.K. Safety and effectiveness of intrauterine devices. Contraception, 11, 243, 1975.

9. MOREHEAD, J.E., MATTHEWS, A., GULLEBAUD, J. \& BONNAR, J., Menstrual blood loss in users of an IUD. An Analysis of intrauterine Contraception (Ed) Hefnawi, F. \& Segal, S.J. p. 381, Amsterdam: North-Holland.1975. 
10. WHITE, M.K., ORTY, H.W. ROOCKS, J.B. \& ROCHAT, R.U. Intrauterine device termination rates and the menstrual cycle day of insertion. Obstetrics and Gynecology. 55 (2). 220. 1980.

11. WHO. Special programm of Research Development and Research training in Human reproduction. Eleventh Annual Report. Geneva, WHO. 159 pp. 1982.

12. WHO. Interval IUD insertion in parous women, a randomised multicentre comparative trial of Lippes loop D.T. Cu 220c and Cooper 7. Contraception, 26, 1-22, 1982.

13. WHO. IUD Insertion following spontaneous abortions clinical trial of the T Cu220, Lippes loop D and Copper 7. Studies in Family planning. 14, 109114, 1983.

14. KOETSAWANG, S., NUKULKARN, P., FOTHERBY, K. Transfer of contraceptive steroids in milk of women using long-acting gestogens. Contraception, 25, 321-331, 1982.

15. MISHELL, D.R., STANCZYK, F., HIROL, M. Steroid contraception. In Ovulation in the Human (Ed). Crosigwani, P.G. \& Mishell, D.R. pp. 141-151., London: Academic Press. 1976.

16. POPULATION REPORTS. Injectables and Implants. Series K. No. 1, K1-16. 1975.

17. WHO Special Programm of Research in Human Reproduction. 10 th Annual Report. p. 42. 1982.
18. WHO TASK Force on Long-Acting Systemic Agents for the regulation of fertility. Multinational comparative clinical evaluation of two long-acting injectable contraceptive steroids; norethisterone enanthate an medroxy progesterone acetate. Contraception. 15, 511 533. 1977.

19. WHO TASK Force on Longe-Acting Agents for the regulation of fertility Multinational comparative clinical trial of long-acting injectable contraceptives. Norethisterone enanthate given in to dosage regimens and depot-medroxy-progesterone acetate. Final report, contraception, 28, 1-20, 1983.

20. FORTNEY, J.A., COLE, L.P. \& KENNEDY, K.I. A new approach to measuring menstrual pattern change after sterilization American Journal of obstetrics and Gynecology, 147, 830-836, 1983.

21. IBRANGKUN, C., PHAOSAVADI, S., NEUWIRTH, R.S. Clinical evaluation of quinacrine hydrochloride for sterilization of the human fermale. Contraception. 14, 75. 1976.

22. KEITH, L.G., \& BERGER, G.S. Culdotomy. In New Trends In sterilization (Ed) Van Lith. d.a.f., Keith, L.G. \& van Hall, E.V. Chicago, London: Year Book Medical, pp. 58. 1983.

24. TATUM H.J. Col. A Decade of intrauterine contraception: 1976-1986. Fertil Steril. Vol. 46: 173-192. 1986. 